Supporting Information

\title{
Solo smart fluorogenic probe for potential cancer diagnosis and tracking in vivo tumorous lymphatic systems via distinct emission signals
}

Soham Samanta, ${ }^{\dagger, a}$ Meina Huang, ${ }^{\dagger, a}$ Fangrui Lin, ${ }^{a}$ Pintu Das, ${ }^{a}$ Bingling Chen, ${ }^{a}$ Wei Yan, ${ }^{a}$ Jia-Jie Chen, ${ }^{b}$ Kunmei Ji, ${ }^{b}$ Liwei Liu, ${ }^{a}$ Junle $\mathrm{Qu}^{\mathrm{a}}$ and Zhigang Yang*a

${ }^{a}$ Key Laboratory of Optoelectronic Devices and Systems of Ministry of Education and Guangdong Province, Center for Biomedical Photonics, College of Physics and Optoelectronic Engineering, Shenzhen University, Shenzhen 518060, China

${ }^{b}$ Department of Biochemistry and Molecular Biology, School of Medicine, Shenzhen University, Shenzhen 518060, China.

${ }^{\dagger}$ Equal contribution

*Corresponding emails: jlqu@szu.edu.cn; zhgyang@szu.edu.cn 
Experimental Section [Synthesis and characterization of the probe L, UV-Vis and fluorescence spectroscopic studies, Detection Limit, Molecular Docking, Cytotoxicity assay, Cell culture]. S3-S6

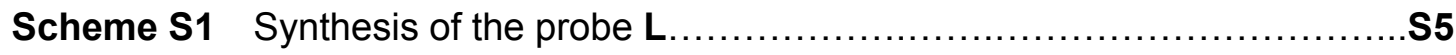

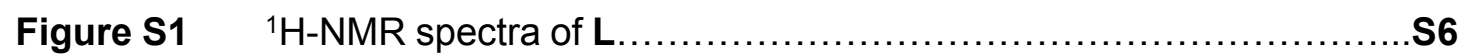

Figure S2 Expanded (aromatic region) ${ }^{1} \mathrm{H}-\mathrm{NMR}$ spectra of L ..................... 6

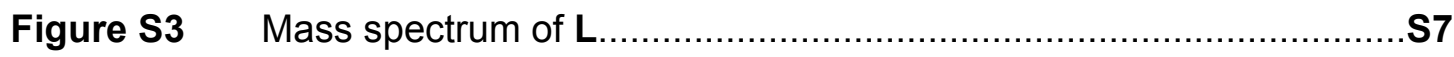

Figure S4 UV-Vis spectra of $\mathbf{L}$ in different solvents............................ 7

Figure S5 Polarity dependent change in fluorescence spectra of $\mathbf{L} \ldots \ldots \ldots \ldots \ldots$. 8

Figure S6 Fluorescence spectra of $\mathbf{L}$ in Dioxane and DMSO ..................S8

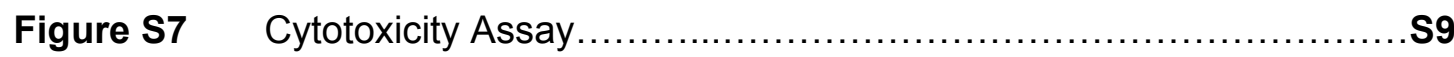

Figure S8 \& S9 Cell imaging with different irradiation time ..................S9-S10

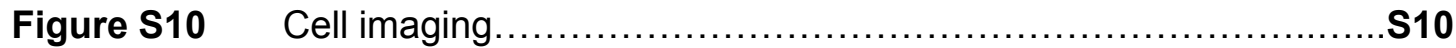

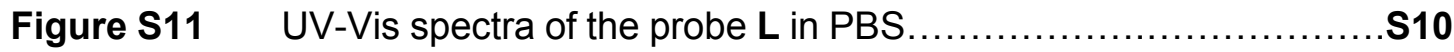

Figure S12 Viscosity dependent changes in fluorescence spectra of

L

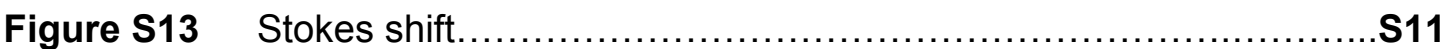

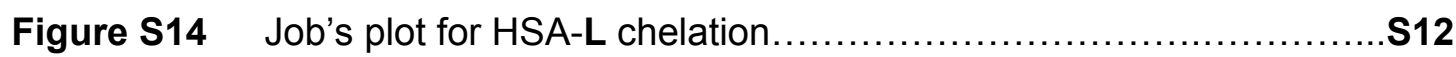

Figure S15 B-H plot for determination of binding constant ....................S12

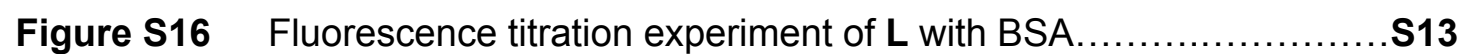

Figure S17 Determination of detection limit................................................... 13

Figure S18 HSA-L interactions obtained from the Docking study.....................S14

Figure S19 Detection of lymph nodes in tumor and normal mice.......................S14

Figure S20 Transportation of the probe towards lymph node............................515

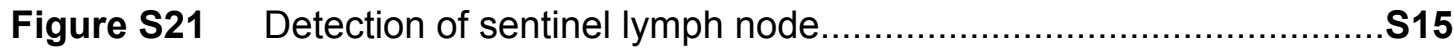

Figure S22 Anatomical study to confirm the position of lymph node..................S16 


\section{Synthesis and characterization of the probe $L$}

In the first step, under a nitrogen atmosphere, $11 \mathrm{mmol}(1.71 \mathrm{~g})$ of (5-formylthiophen-2-yl)boronic acid and the $10 \mathrm{mmol}(3.24 \mathrm{~g})$ of 4-Bromotriphenylamine compound were dissolved in $35 \mathrm{~mL}$ of dioxane. To the mixture $0.55 \mathrm{mmol}$ of $\mathrm{Pd}\left(\mathrm{PPh}_{3}\right)_{4}$ and $20 \mathrm{mmol}$ of $\mathrm{Na}_{2} \mathrm{CO}_{3}$ (as $5 \mathrm{~mL}$ aqueos solution) were added. The whole reaction mixture was then refluxed with continous stirring under the nitrogen atmosphere for $20 \mathrm{~h}$. A brown-black reaction mixture was evident. The reaction mixture was then cooled to room temperature and filtered. To the filtrate, $\mathrm{DCM}(50 \mathrm{~mL})$ and water $(30 \mathrm{~mL})$ were added and shaken well. The organic layer was separated and dried over the $\mathrm{MgSO}_{4}$. The sovent was evaporated under the reduced pressure and the crude was subjected to the column chromatography (eluent EtOAc/Hexane in the ratio of $5: 95)$ to obtain the pure yellow solid product of 5-(4-(diphenylamino)phenyl)thiophene-2-carbaldehyde (Step 1, Sheme S1).

In the second step, $10 \mathrm{mmol}(1.49 \mathrm{~g})$ of 2-Methylbenzothiazole and $12 \mathrm{mmol}$ of iodomethane $(1.69 \mathrm{~g})$ were taken in a round bottomed flask containing $20 \mathrm{~mL}$ of acetonitrile. The reaction mixture was then refluxed with continous stirring for $12 \mathrm{~h}$. A white solid product, appeared in the reaction mixture, was then filtered and washed with cold methanol to get 2,3-dimethylbenzothiazol-3-ium iodide (Step 2, Sheme S1). The product was then dried in open air.

In the final step, $1.0 \mathrm{mmol}$ of the product obtained from the first step [5-(4-(diphenylamino)phenyl)thiophene-2-carbaldehyde] and $1 \mathrm{mmol}$ of the product, obtained in the second step (2,3-dimethylbenzothiazol-3-ium iodide) were dissolved in dry $\mathrm{EtOH}$. The resultant mixture was then refluxed for $12 \mathrm{~h}$ to yield the dark pink-red colour product of $\mathbf{L}$. The product was filtered, washed with cold methanol three times and dried in a desiccator.

Calculated yield (for the final step): $71 \% .{ }^{1} \mathrm{H}$ NMR [500 MHz, DMSO-d 6 , J (Hz), $\delta$ (ppm)]: 8.43-8.38 $(2 \mathrm{H}, \mathrm{m}), 8.20(1 \mathrm{H}, \mathrm{d}, J=8.0), 7.94(1 \mathrm{H}, \mathrm{d}, J=4.0), 7.86(1 \mathrm{H}, \mathrm{t}, J=$ 7.0), $7.77(1 \mathrm{H}, \mathrm{t}, J=8.0), 7.69(2 \mathrm{H}, \mathrm{d}, J=9.0), 7.65(1 \mathrm{H}, \mathrm{d}, J=4.0), 7.61(1 \mathrm{H}, \mathrm{d}, J=$ 15.5), $7.39(4 \mathrm{H}, \mathrm{t}, J=8.0), 7.16-7.10(6 \mathrm{H}, \mathrm{m}), 6.99(2 \mathrm{H}, \mathrm{d}, J=8.5), 4.29(3 \mathrm{H}, \mathrm{s})$. ESI-MS (positive mode, m/z) Calculated for $\mathrm{C}_{32} \mathrm{H}_{25} \mathrm{~N}_{2} \mathrm{~S}_{2}+$ : 501; Found: 501 .

\section{UV-Vis and fluorescence spectroscopic studies}

Stock solution of $\mathbf{L}\left(1 \times 10^{-3} \mathrm{~mol} \mathrm{~L}^{-1}\right)$ was prepared in DMSO. It was diluted to $2 \times 10^{-6}$ mol $\mathrm{L}^{-1}$ for various spectral studies by placing only $4.0 \mu \mathrm{L}$ of the stock solution of $\mathrm{L}$ into an aqueous phosphate-buffered saline (PBS) solution $(\mathrm{pH} 7.4)$ to a final volume of $2.0 \mathrm{~mL}$. Stock solutions of $\mathrm{HSA}$ and $\mathrm{BSA}(20 \mathrm{mg} / \mathrm{mL})$ were prepared in phosphate-buffered saline (PBS) solution ( $\mathrm{pH} 7.4)$. The stock solutions of other proteins and bioanalytes were prepared as necessary, and the concentrations of the protein stock solutions were calculated from the molar-extinction coefficient values at particular wavelengths as relevant. In the fluorescence selectivity experiment, the test samples were prepared by adding the appropriate amounts of the stock solutions of the respective proteins or analytes into $2.0 \mathrm{~mL}$ of probe solution $\left(2 \times 10^{-6} \mathrm{~mol} \mathrm{~L}^{-1}\right)$. For the fluorescence-titration experiments, another set of HSA standard solutions was prepared with $5 \mathrm{mg} / \mathrm{mL}$ concentrations by diluting the earlier prepared stock solution 
(20 mg/mL). Quartz optical cells with $1.0 \mathrm{~cm}$ path lengths were then filled with $2.0 \mathrm{~mL}$ solutions of $\mathbf{L}$ for the fluorescence-titration experiment, to which the newly prepared stock solutions of HSA $(5 \mathrm{mg} / \mathrm{mL})$ were gradually added using a micropipette as required. For the fluorescence experiments, excitation was set at $490 \mathrm{~nm}$ and emission was acquired from 520 to $850 \mathrm{~nm}$ except for the polarity and viscosity dependent studies. Particularly, for the solvent polarity dependent fluorescence spectral studies, the excitation was provided at $420 \mathrm{~nm}$ and emission was scanned from 440 to $800 \mathrm{~nm}$. All the spectral data were recorded within $1 \mathrm{~min}$ after the addition of the analytes unless mentioned otherwise.

\section{Detection Limit}

The detection limits was calculated on the basis of the fluorescence titration with BSA. The fluorescence emission spectrum of $\mathbf{L}$ was measured 10 times to calculate the standard deviation (at $673 \mathrm{~nm}$ ) of blank measurement. Thereafter, the fluorescence emission at $673 \mathrm{~nm}$ was plotted as a function of the concentration of BSA from the corresponding titration experiment to evaluate the slope.

The detection limit was then calculated using the following equation:

Detection limit $=3 \sigma / \mathrm{k}$.

where $\sigma$ is the standard deviation of blank measurement, and $k$ is the slope between the fluorescence emission intensity versus [BSA].

\section{Molecular Docking}

To observe the geometrically and energetically most stable conformation of the probe L when it binds with HSA, the Molecular docking study was performed. AutoDockTools-1.5.6 was used to perform the docking study and achieve the conformations of L-HSA adduct. The binding free energies and binding sites were determined using this software. The crystal structure of HSA was downloaded from RCSB PDB (4K2C) (https://www.rcsb.org/structure/4K2C). ${ }^{\text {s1 }}$ A grid box was generated with a spacing of $0.508 \AA$ and dimensions of $90 \times 70 \times 90$ points (.gpf file) to run the autogrid. For running autodock, the output was selected as a Lamarckian GA (.dpf file). The docking results were analyzed and the conformation with lowest binding energy (among the 10 conformations) has been saved as result and opened in the PyMOL viewer programs to obtain the docking structure with lowest binding energy.

\section{Cytotoxicity assay}

Cytotoxicity assays were carried out using HeLa cells. Cell viability was determined using Cell Counting Kit-8 (CCK8) assay. 10000 cells per well were seeded in a 96-well plate and incubated in a humidified incubator for adherence. After $24 \mathrm{~h}$ of culturing, the medium in each well was replaced by $100 \mu \mathrm{L}$ of fresh medium (RPMI-1640) containing different concentrations $(0,2,4,6,8 \mu \mathrm{M})$ of the probe $\mathrm{L}$. The solution was filtrated by $0.22 \mu \mathrm{m}$ sterile filter. The volume fraction of DMSO was below $0.2 \%$. $24 \mathrm{~h}$ later, CCK-8 reagent, diluted by RPMI-1640 medium (10\%) was added to each well after the removal of culture media and incubated for $0.5 \mathrm{~h}$. Following that, 
the absorbance was measured at $450 \mathrm{~nm}$ on a plate reader (RT-6100, Rayto Life and Analytical Sciences Co., Ltd, Shenzhen, China). Each trial was performed with six parallel wells. Cell viability rate was determined as VR $=(A s-A b) /(A c-A b) \times 100 \%$, where As is the absorbance of the experimental group, Ac is the absorbance of the control group (no probe $L$ ), and $A b$ is the absorbance of the blank group (no cells).

\section{Cell culture}

Both cancer cells (4T1 and HeLa) as well as normal cells (3T3 and COS-7) were utilized in this work. The 4T1 cells and HeLa cells were cultured in RPMI-1640 medium and DMEM medium was used for 3T3 cells and COS-7 cells. All culturing media were supplemented with 10\% (v/v) FBS (gibco) and $1 \%$ antibiotics (10000 Units $/ \mathrm{mL}$ penicillin and $10000 \mu \mathrm{g} / \mathrm{mL}$ streptomycin, gibco) under $5 \% \mathrm{CO}_{2}$ atmosphere and $95 \%$ humidity at $37^{\circ} \mathrm{C}$.

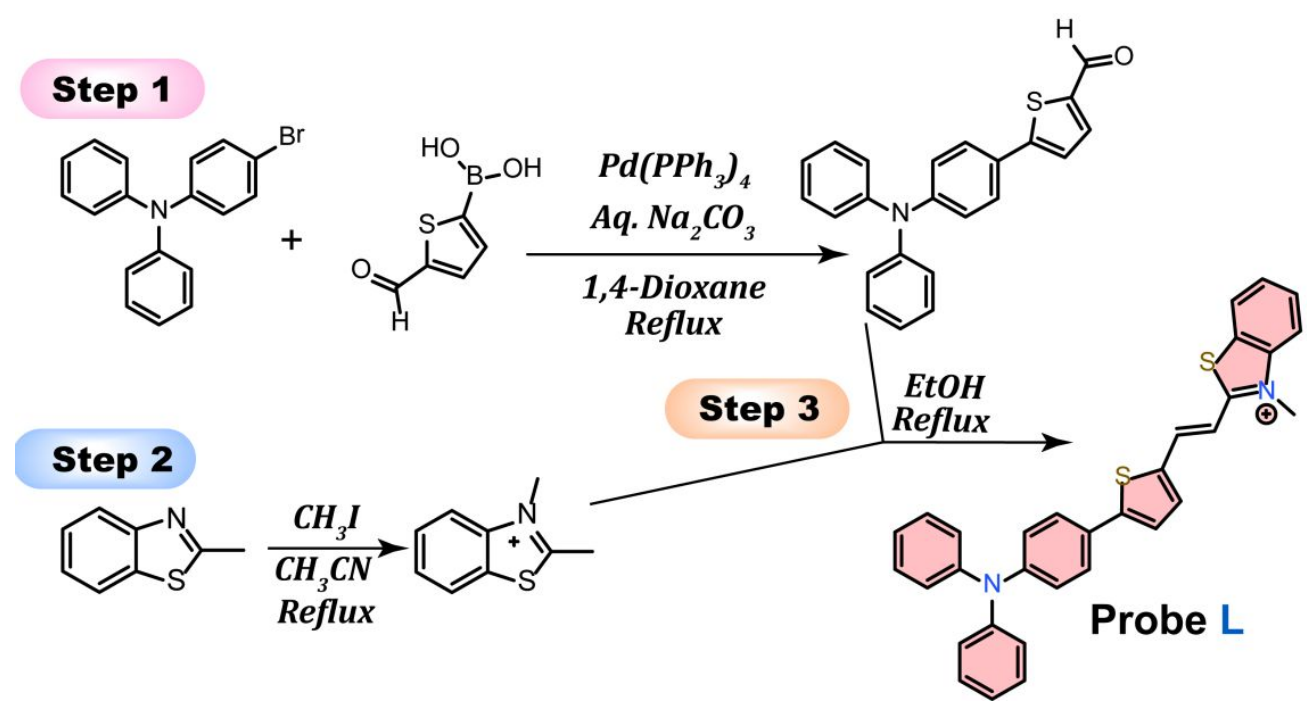

Scheme S1. Synthetic scheme of the probe $\mathbf{L}$ 


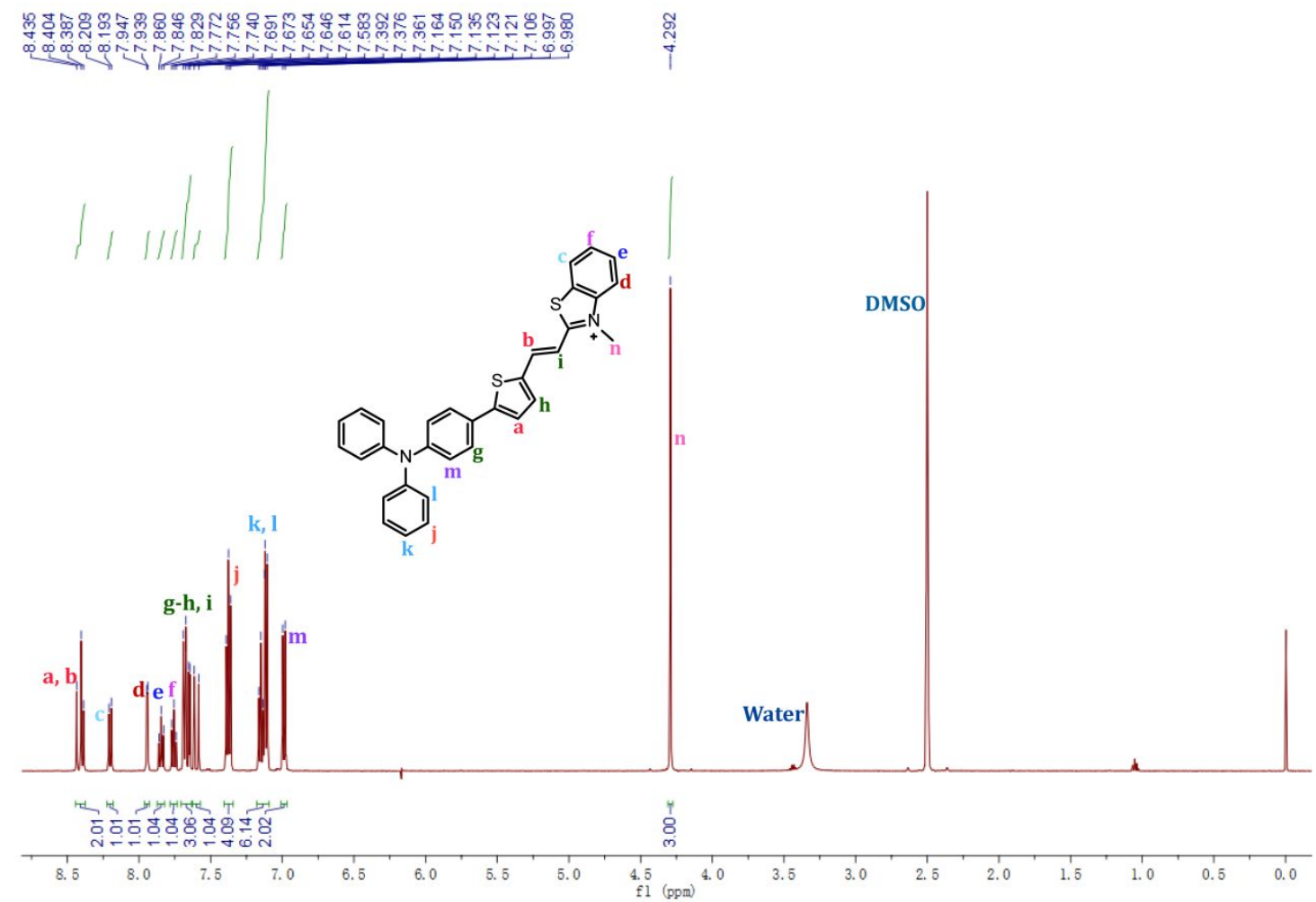

Figure S1: ${ }^{1} \mathrm{H}$ NMR spectra of $L$ in $\mathrm{DMSO}^{-\mathrm{d}_{6}}$.

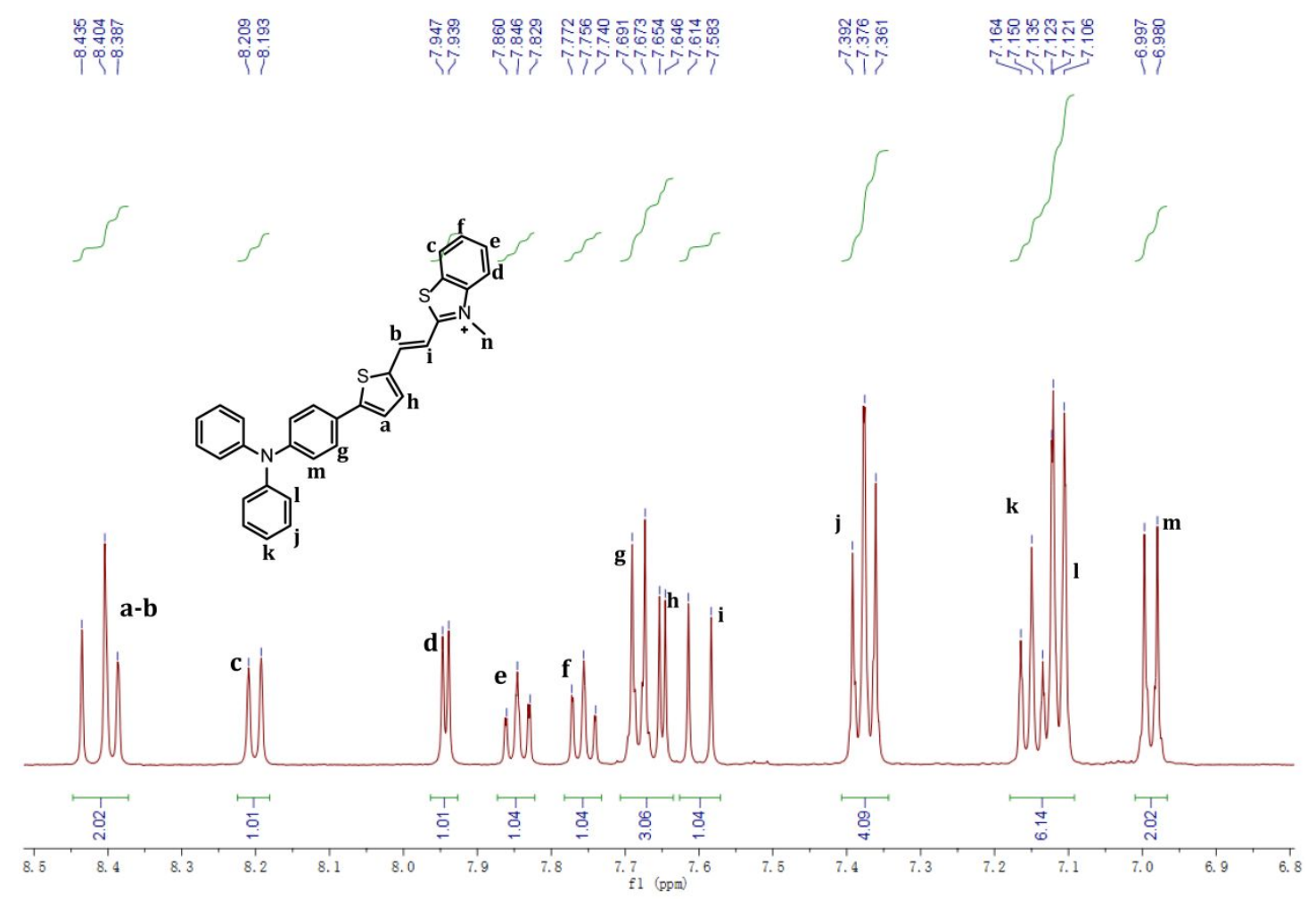

Figure S2: Expanded aromatic region of the ${ }^{1} \mathrm{H}$ NMR spectra of $\mathbf{L}$ in DMSO-d . $_{\text {. }}$ 


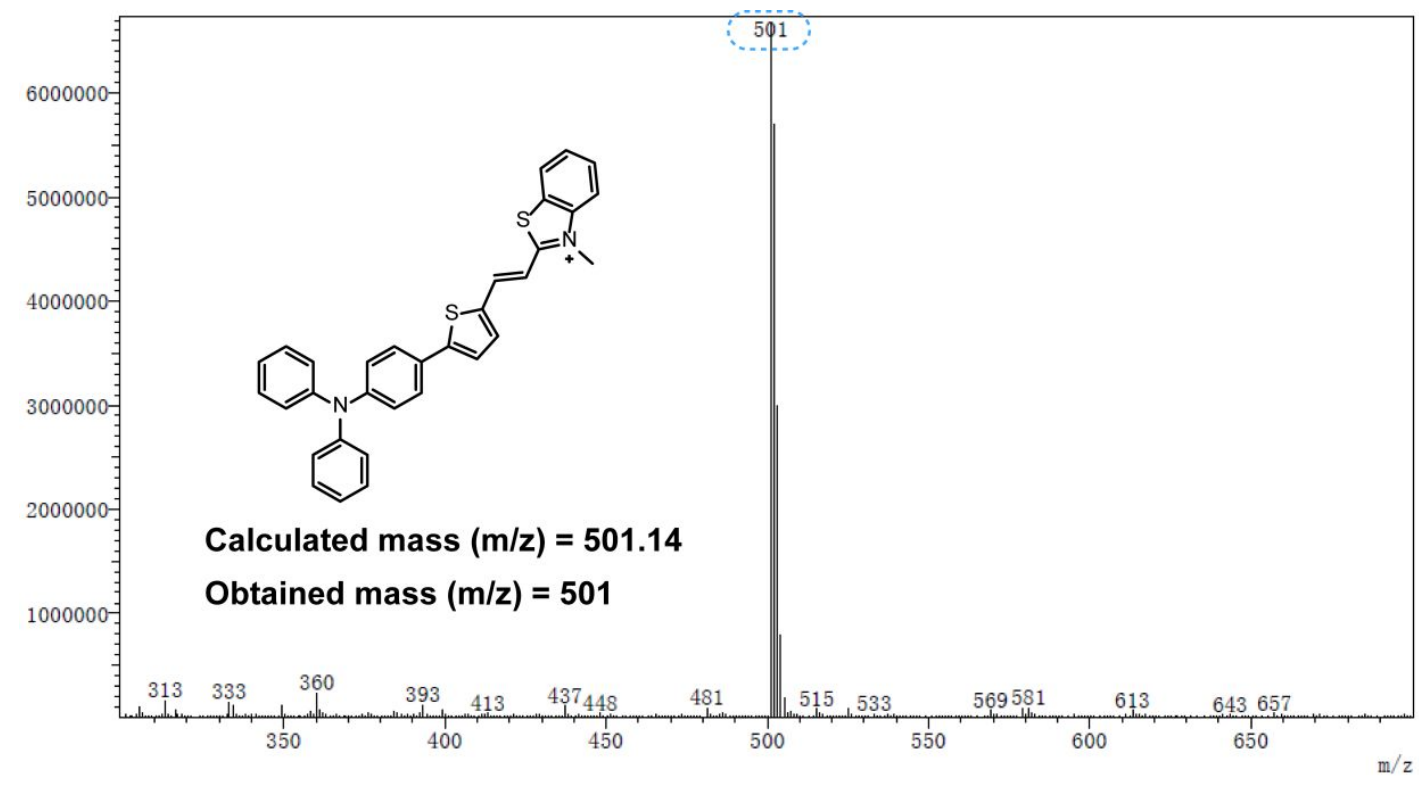

Figure S3: Mass spectrum of $\mathbf{L}$.

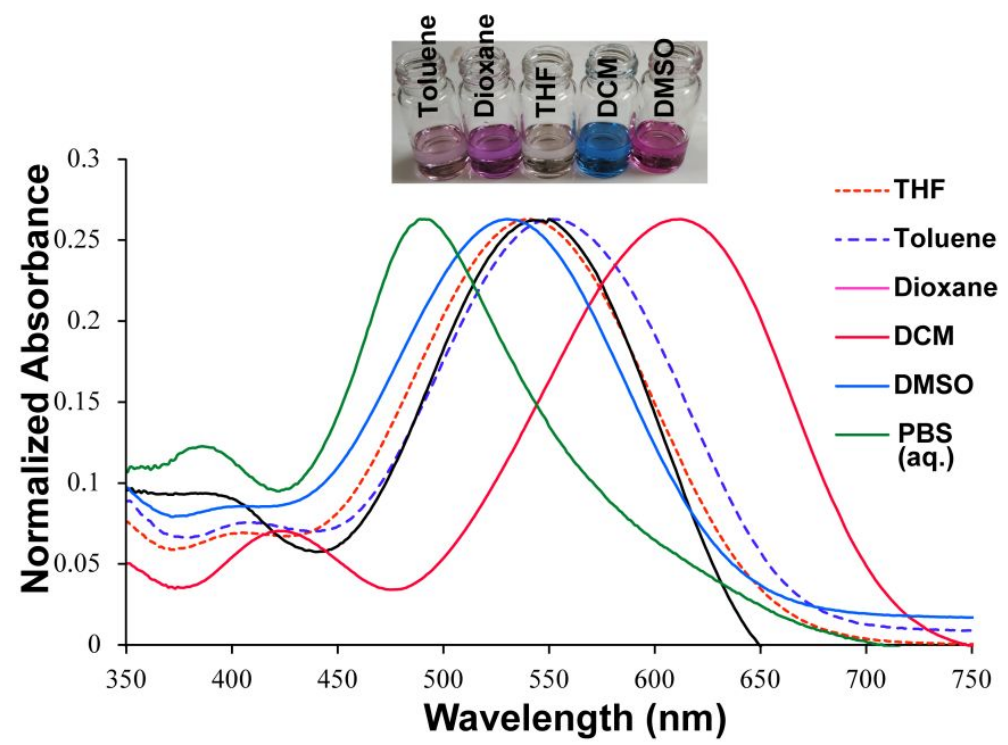

Figure S4: Changes in the UV-Vis spectra of $L(10 \mu \mathrm{M})$ in different solvents with diverse polarity; inset: visual changes in the color of the solutions of $\mathbf{L}$ in different solvents. 


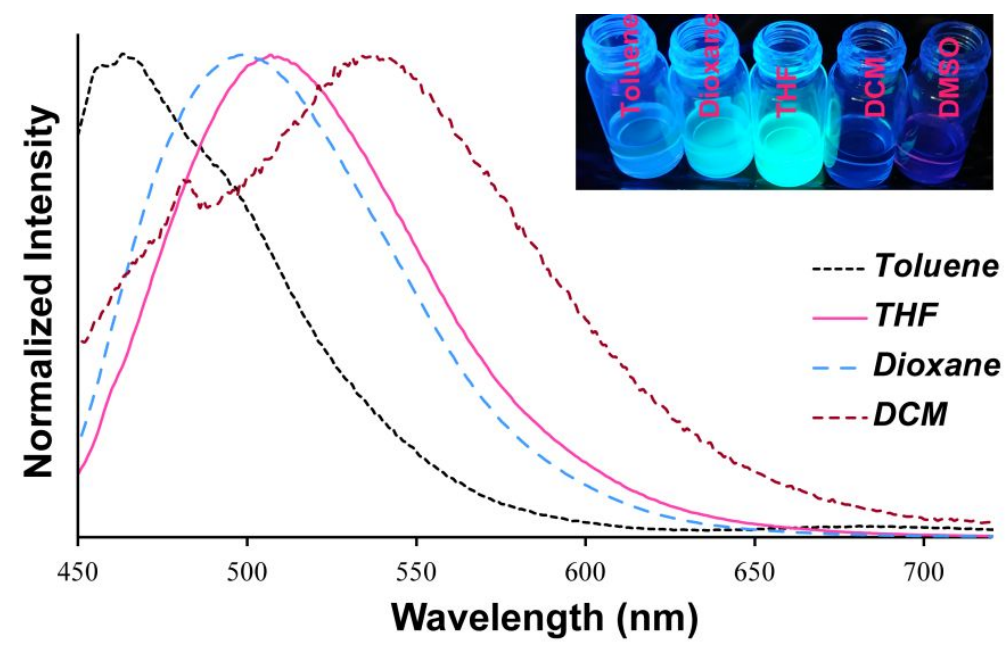

Figure S5: Shifts in the emission maxima of $L(2 \mu \mathrm{M})$ upon changing the solvent polarity; $\lambda_{\mathrm{ex}}=430 \mathrm{~nm}$.

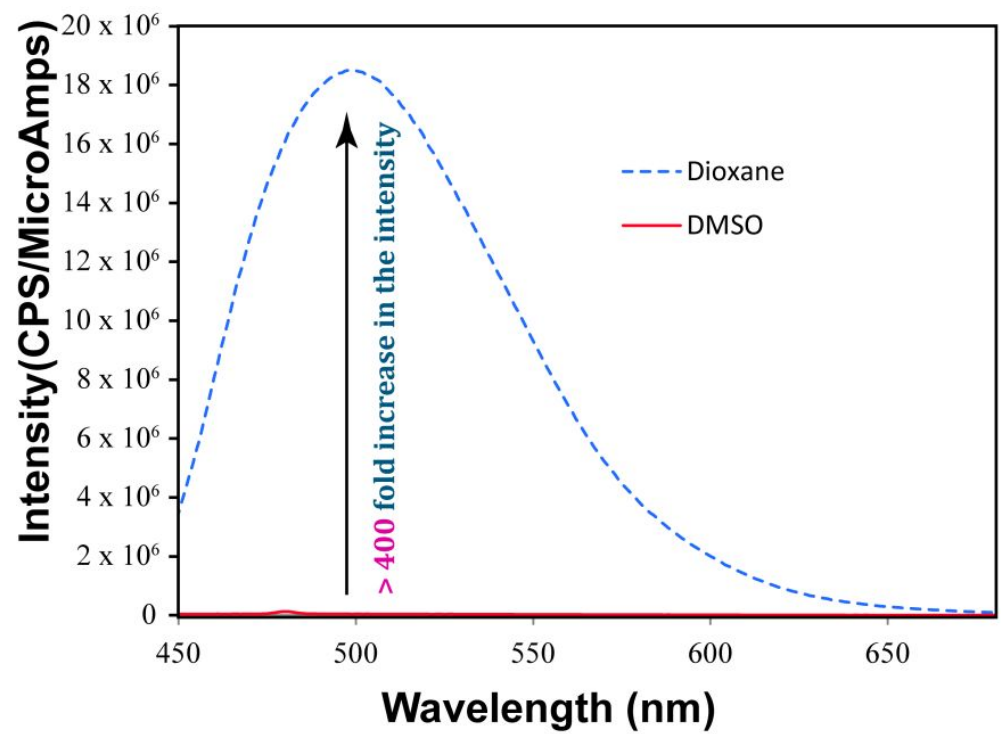

Figure S6: Fluorescence spectra of $L(2 \mu \mathrm{M})$ in Dioxane and DMSO at the same experimental condition; $\lambda_{\mathrm{ex}}=430 \mathrm{~nm}$. 


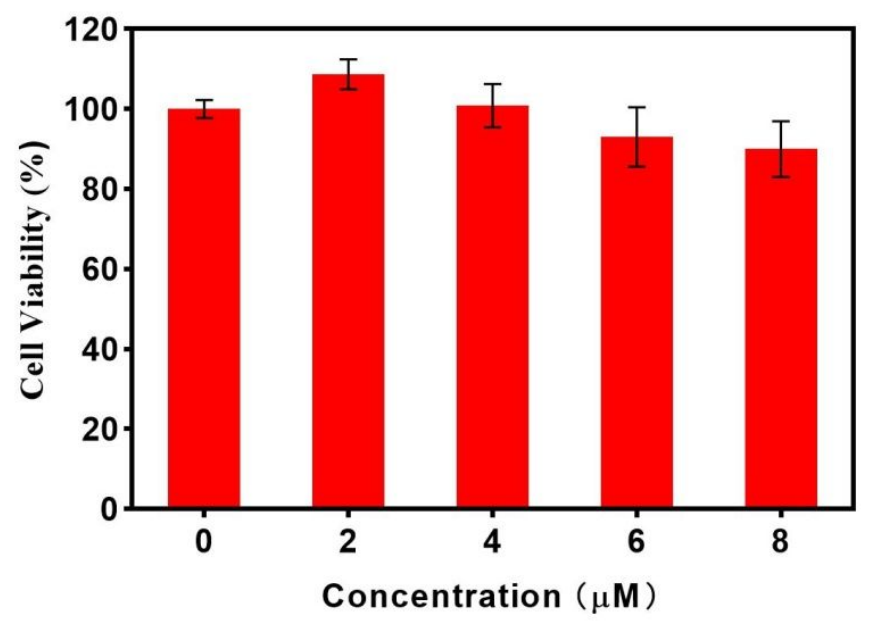

Figure S7: CCK-8 cytotoxicity assay for the probe $\mathbf{L}$.

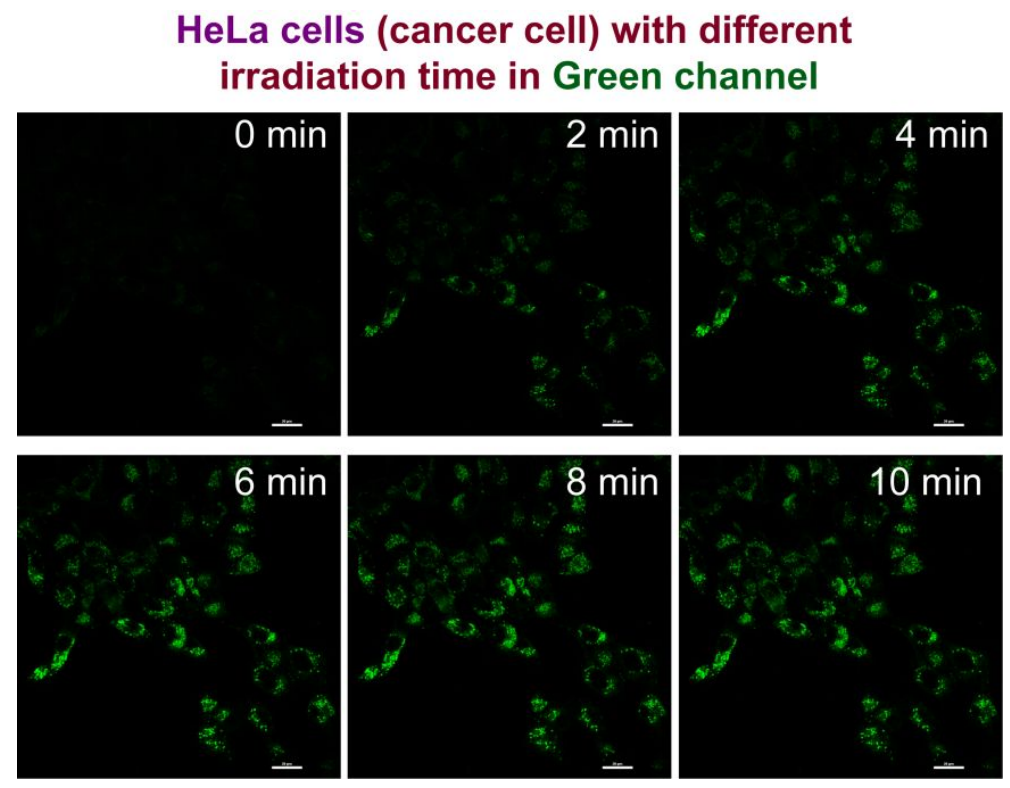

Figure S8: Fluorescence imaging of the HeLa cells, incubated with the probe $\mathbf{L}$ in different irradiation time in the imaging instrument; $\lambda_{\mathrm{ex}}=488 \mathrm{~nm}$ and emission range: $500-550 \mathrm{~nm}$. Scale bar $20 \mu \mathrm{m}$. 


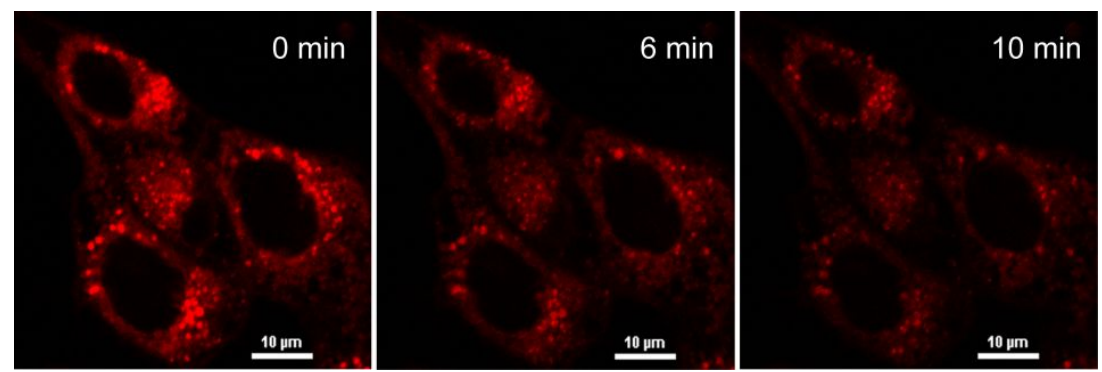

Figure S9: Fluorescence imaging of the probe $\mathbf{L}$ incubated HeLa cells in different irradiation time in the instrument; $\lambda_{\mathrm{ex}}=561 \mathrm{~nm}$ and emission range: 663-736 nm.
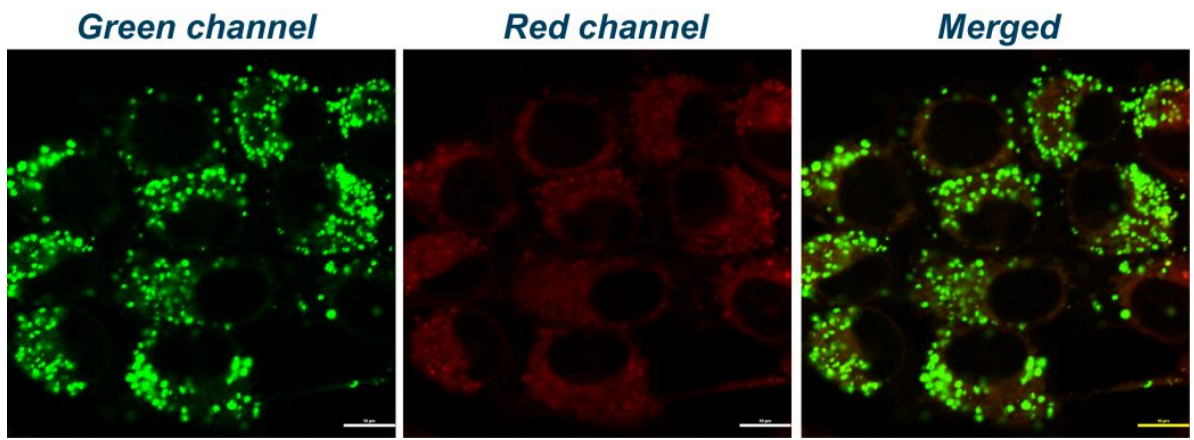

Figure S10: Confocal fluorescence microscopy images of the cancer cells (4T1 cells) incubated with $3 \mu \mathrm{M}$ of $\mathbf{L}$ for $30 \mathrm{~min}$ in green (excitation $488 \mathrm{~nm}$ ) and red emission (excitation $561 \mathrm{~nm}$ ) channels (after 6 min irradiation). The scale bar is $10 \mu \mathrm{m}$.

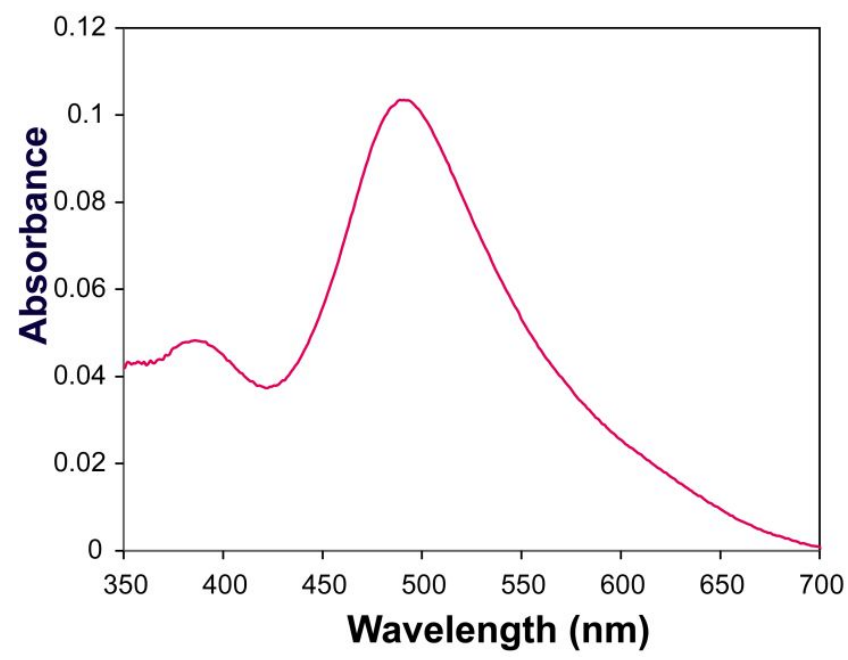

Figure S11: UV-Vis spectra of the probe $\mathbf{L}(10 \mu \mathrm{M})$ in aqueous PBS buffer (pH 7.4). 


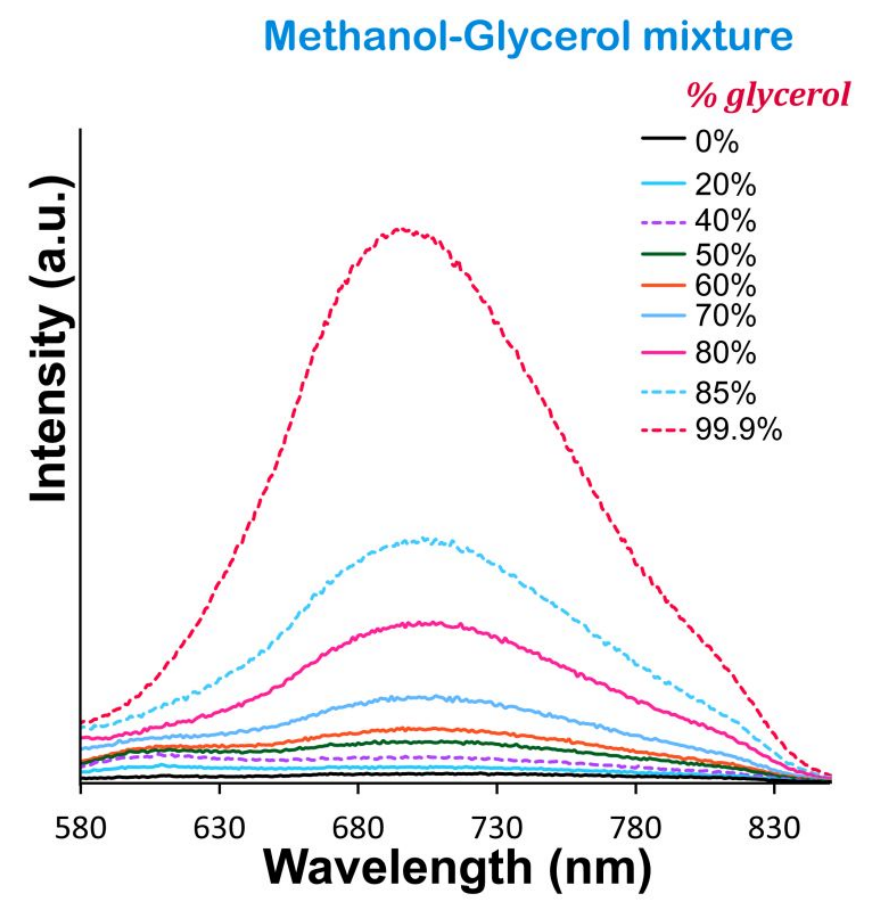

Figure S12: Fluorescence spectra of $L(2 \mu \mathrm{M})$ in different ratios of methanolglycerol mixtures representing fluorescence changes with varying viscosity.

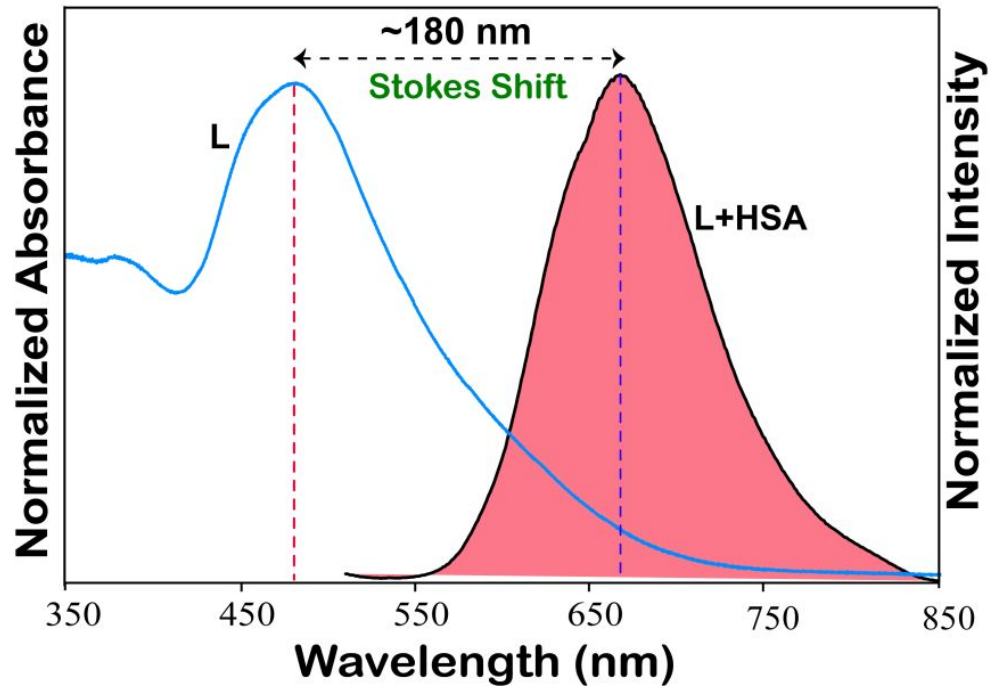

Figure S13: Stokes shift of the probe $L(2 \mu M)$ for HSA sensing. 


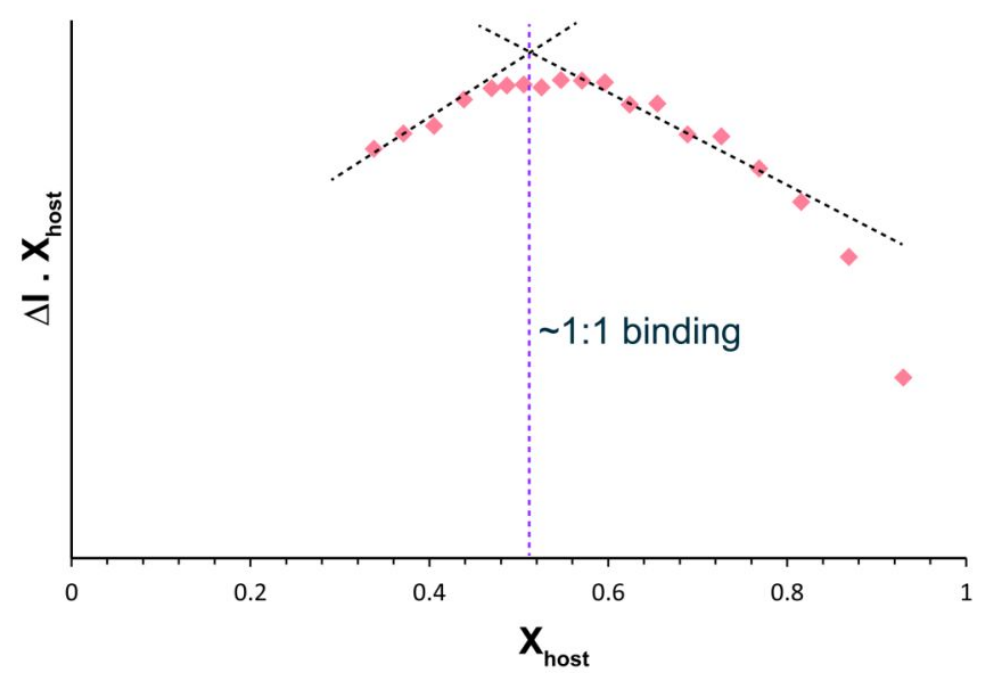

Figure S14: Job's plot obtained from the fluorescence titration experiment showing 1:1 chelation between $\mathbf{L}$ and HSA.

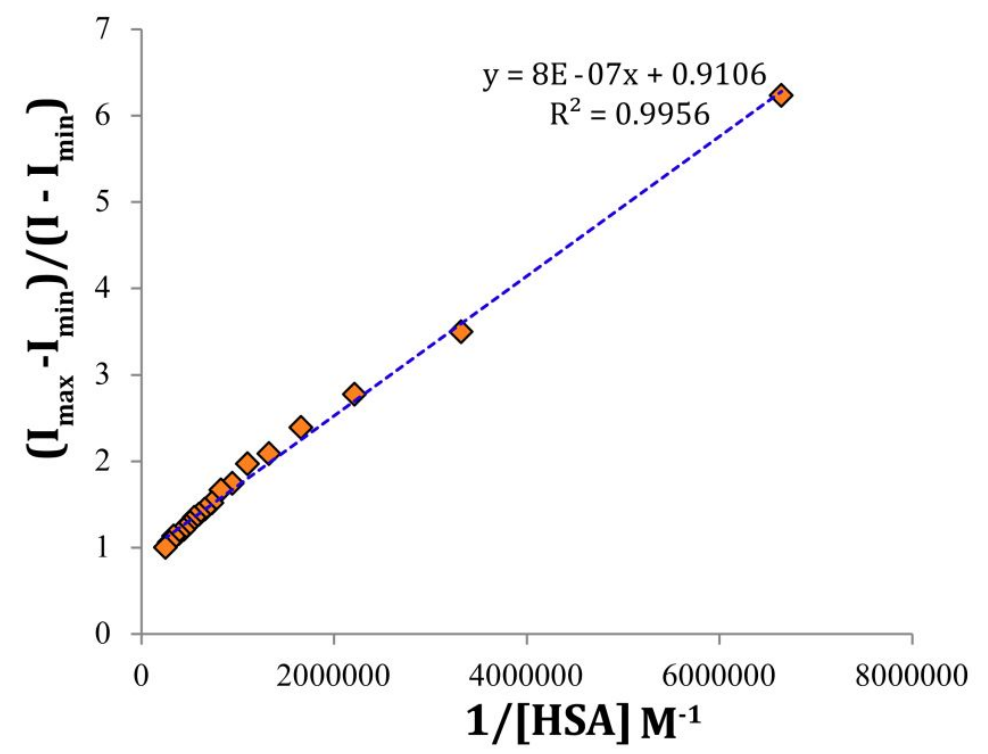

Figure S15: B-H plot for determination of binding constant (L-HSA complex). 


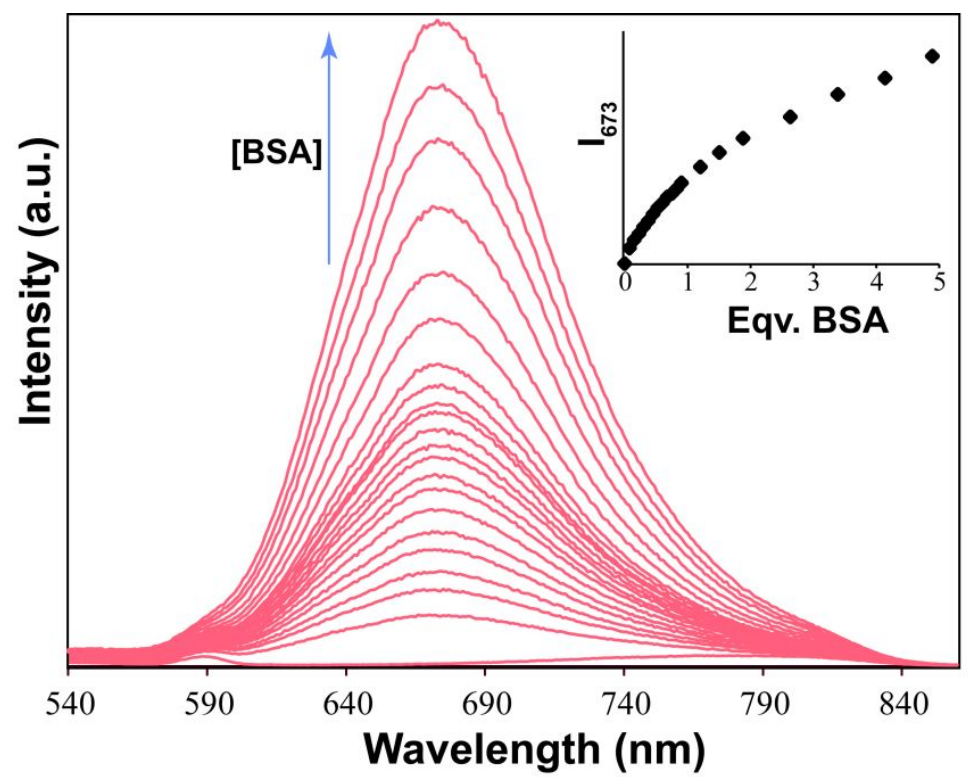

Figure S16: Fluorescence titration with the probe $\mathbf{L}$ in presence of different concentrations of BSA. $\lambda_{\mathrm{ex}}=490 \mathrm{~nm}$

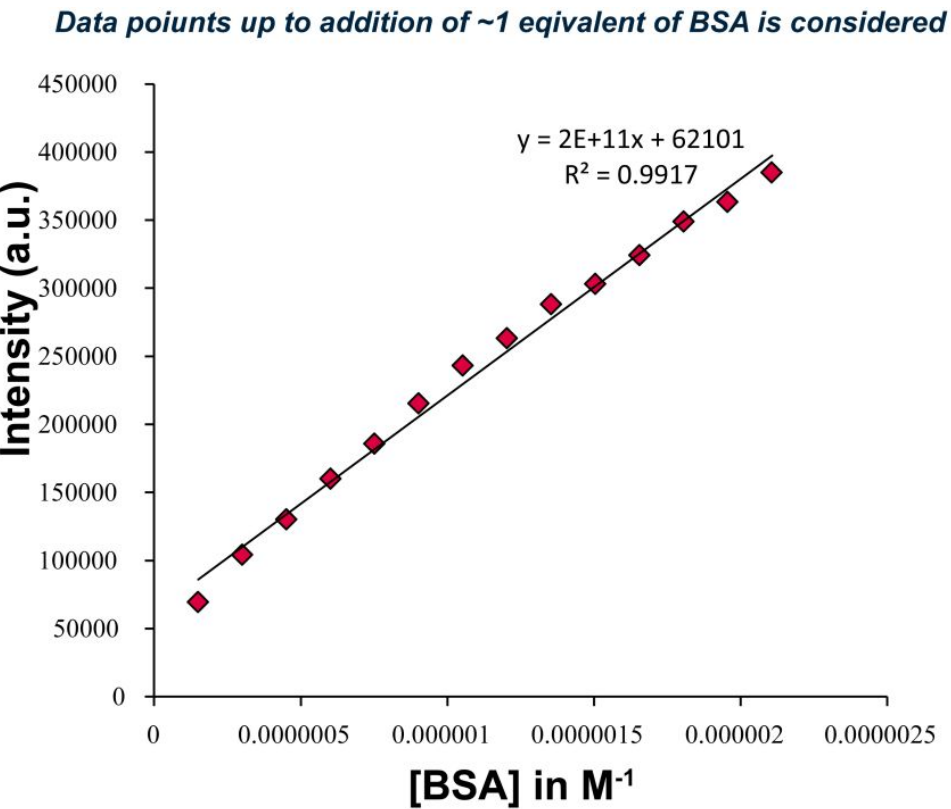

Figure S17: Fluorescence intensity (at $673 \mathrm{~nm}$ ) vs. concentration of BSA plot for determination of detection limit. 


\begin{tabular}{|c|c|c|c|c|c|}
\hline Index & Residue & AA & Distance & Ligand Atom & Protein Atom \\
\hline 1 & $114 \mathrm{~A}$ & ARG & 3.99 & 5831 & 1072 \\
\hline 2 & $115 \mathrm{~A}$ & LEU & 3.45 & 5644 & 1091 \\
\hline 3 & $115 \mathrm{~A}$ & LEU & 3.67 & 5622 & 1088 \\
\hline 4 & $138 \mathrm{~A}$ & TYR & 3.86 & 5845 & 1316 \\
\hline 5 & $138 \mathrm{~A}$ & TYR & 3.68 & 5844 & 1318 \\
\hline 6 & $141 \mathrm{~A}$ & GLU & 3.74 & 5845 & 1353 \\
\hline 7 & $142 \mathrm{~A}$ & ILE & 3.69 & 5844 & 1384 \\
\hline 8 & $181 \mathrm{~A}$ & TYR & 3.35 & 5816 & 1577 \\
\hline 9 & $161 \mathrm{~A}$ & TYR & 3.86 & 5817 & 1575 \\
\hline 10 & $182 \mathrm{~A}$ & LEU & 3.28 & 5616 & 1763 \\
\hline 11 & $185 \mathrm{~A}$ & LEU & 3.24 & 5817 & 1788 \\
\hline
\end{tabular}

\begin{tabular}{l|lllllll|l}
\hline In-Stadking & $\ldots . .$. &... \\
\hline 1 & 138A & TYR & 5.29 & 69.47 & 1.55 & T & $5613,5614,5615,5616,5617,5618$ \\
\hline
\end{tabular}

T-Cation Interactions ....
\begin{tabular}{|llllllll|}
\hline Index & Residue AA & Distance & Offset & Protein charged? & Ligand Group Ligand Atoms \\
\hline 1 & $114 \mathrm{~A}$ & ARG & 3.98 & 1.62 & $\checkmark$ & Aromatic & $5626,5627,5628,5629,5630$ \\
\hline
\end{tabular}

Figure S18: Interactions, encountered in the most stable Docking conformation of L-HSA complex (obtained from Protein-Ligand Interaction Profiler PLIP).

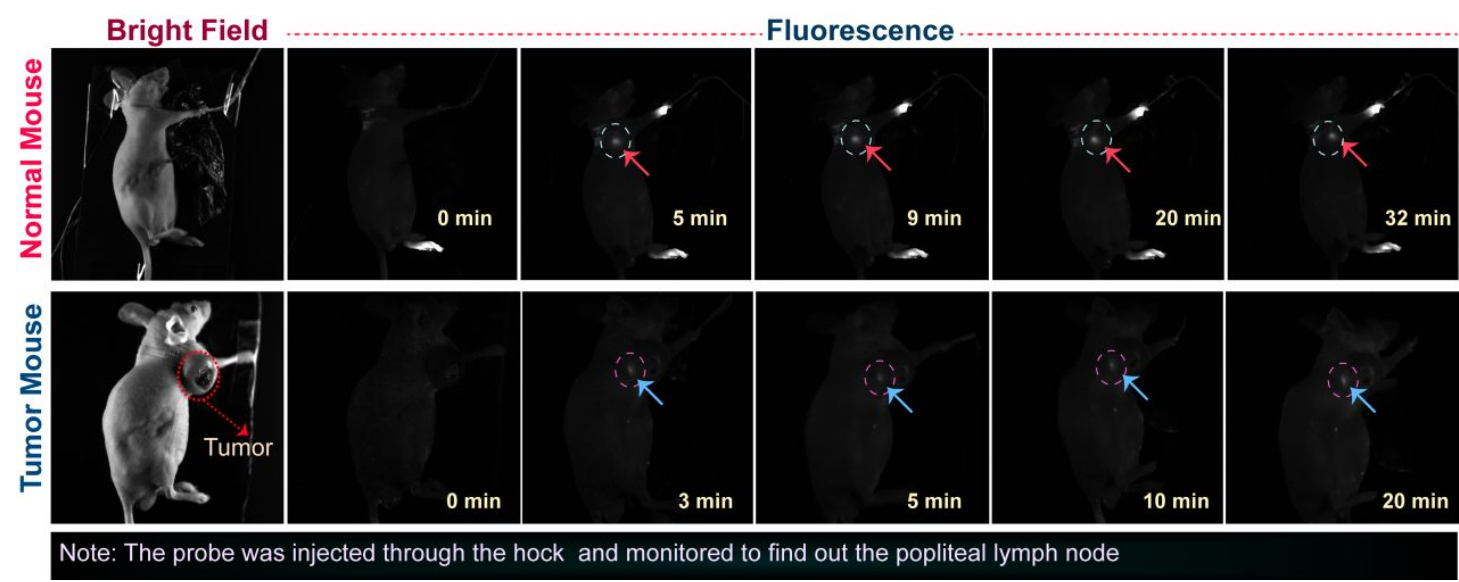

Figure S19: Detection of the lymph node(s) when the probe is injected from the hock/right foot pad in normal and tumor-bearing mice. 

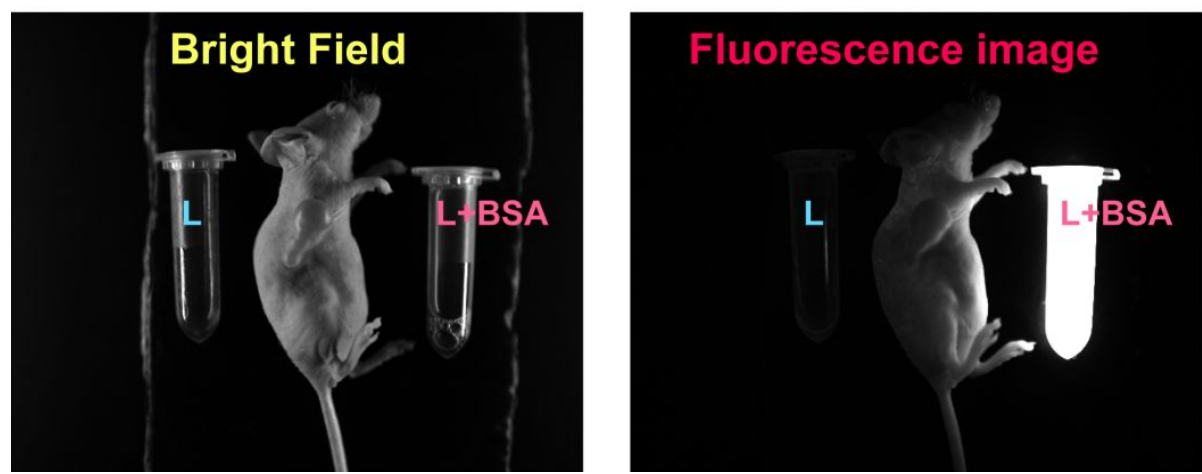

Figure S20: Blank study with $L$ and $L+B S A$ in the in vivo imaging instrument.

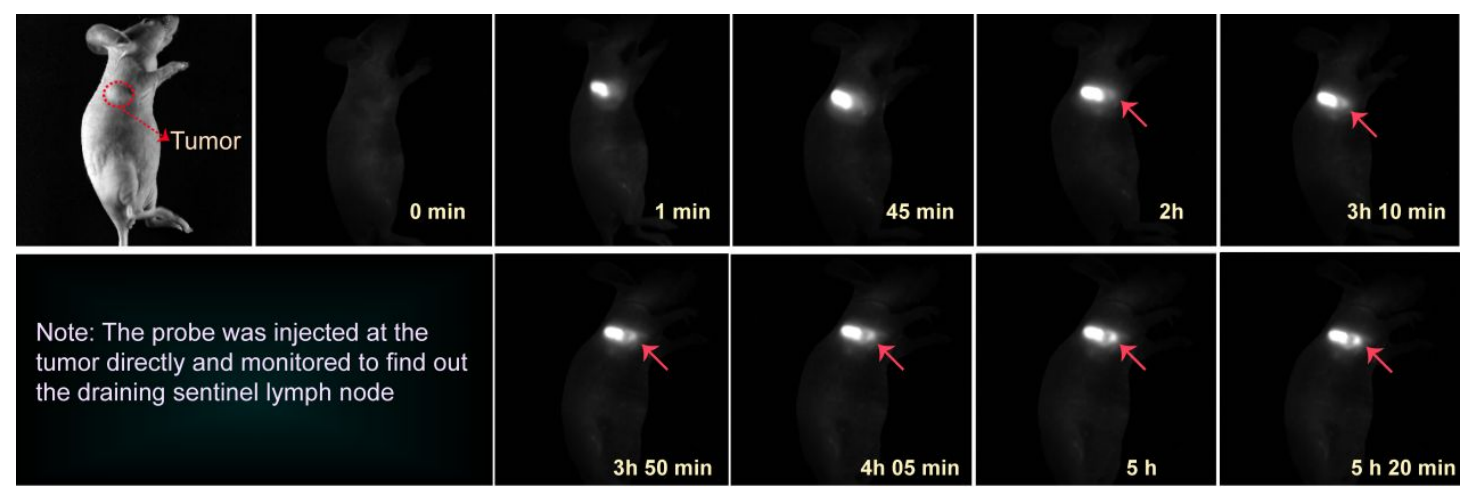

Figure S21: Monitoring the sentinel lymph node (SLN) with the fluorescent probe $\mathbf{L}$ with time when injected from the tumor itself in a tumor-bearing mouse. 


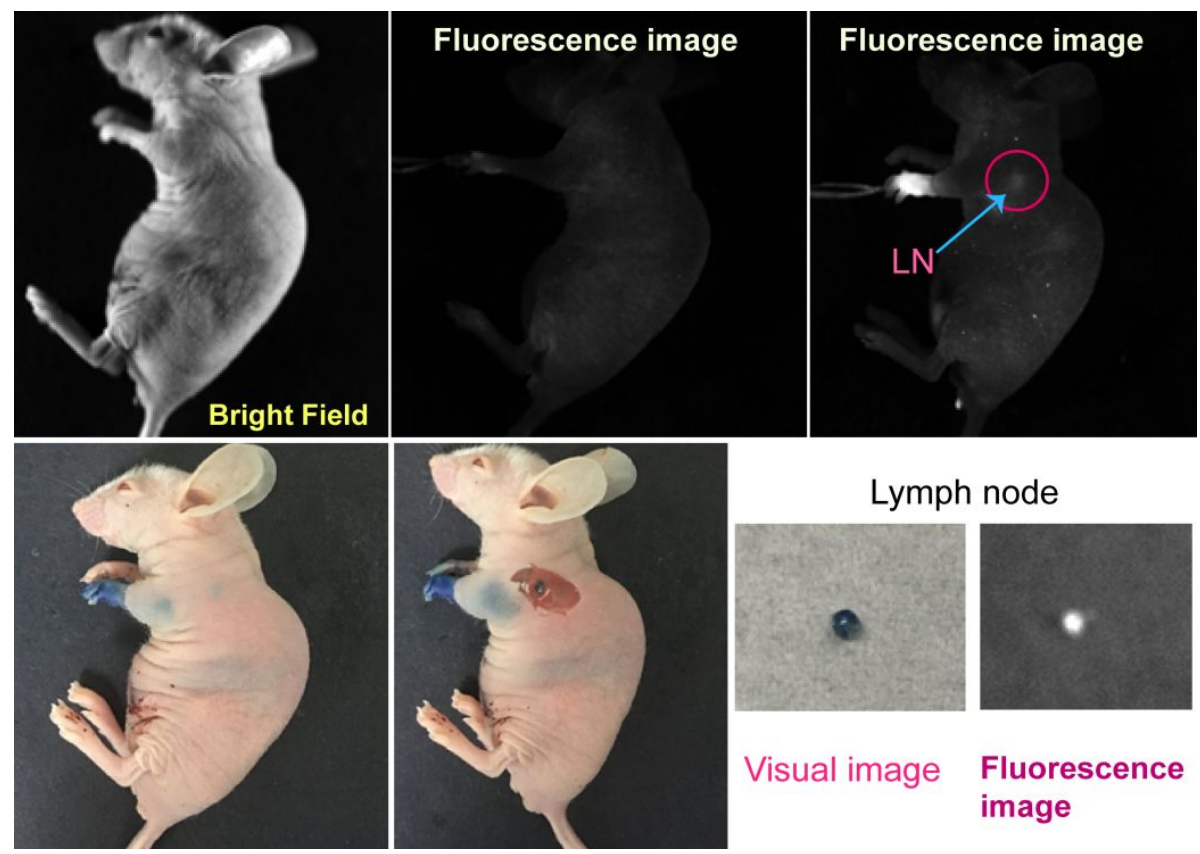

Figure S22: Anatomical study to confirm the position of lymph node in case of normal mouse.

\section{Reference.}

S1. Wang, Y., Yu, H., Shi, X, Luo, Z., Lin, D., Huang, M. Structural Mechanism of Ring-Opening Reaction of Glucose by Human Serum Albumin. J. Biol. Chem. 2013, 288, 15980-15987. 